\title{
Efficacy of Facts in Power Oscillation Damping and Renewable Integration
}

\author{
Alok Pratap Singh ${ }^{1}$ Annewsha Datta ${ }^{2}$ Chandan Mallick $^{3}$ Saugato Mondal ${ }^{4}$ \\ ${ }^{1}$ Department of Electrical Engineering I.I.T (BHU),Varanasi \\ ${ }^{2}$ Department of Electrical Engineering , NSEC, Kolkata \\ ${ }^{3}$ Department of Electrical Engineering,I.I.T Dhanbad \\ ${ }^{4}$ Department of Electrical Engineering ,IIEST Shibpur, Kolkata
}

\begin{abstract}
This paper discusses about the approach towards effective power oscillation damping, root causes for the genesis of power oscillation and various methodologies adopted to eradicate it.This paper presents an unified approach of FACTS controller installation in order to ensure effective power oscillation damping. The power oscillations, if not controlled within time, may aggravate the entire power system's functioning and ultimate outcome as blackout. The network congestion has been a matter of serious concern to utility operations due to classical transmission corridors and inadequate controllers' deployment. The necessity of system regulations demands an adequate controller for matching the power requirement variability and this has been demonstrated with STATCOM control feature. This controller enhances the Available Transfer Capability along with security and reliability. IEEE 14 Bus system is simulated and studied with various scenarios with the help of MATLAB and PSAT to identify various complexities and to resolve these issues so as to enhance the ATC, reliability and security
\end{abstract}

Keywords: Available Transfer Capability (ATC), Flexible A C transmission (FACTS), power system stabilizer (PSS), UPFC.

\section{Introduction}

The power system operation has been very complex due to massive transmission corridor length and connectivity of multi-power injection units and loads connectivity. The system operators find very difficult to manage power flow during peak hours due to poor network availability and required ATC. The loading pattern keeps on changing over the 24 hours due to dynamical requirements of the consumers. The long transmission network corridor sometimes fail to evacuate due to constrained ATC which may result in dip in the voltage and followed by frequency variation at the interface boundary and finally the areas connected may be separated by protection devices just to safe the entire network by SPS. Under such a situation, the strategic deployment of FACTS which may result not only to enhance the ATC but also quick power oscillation damping and thus ensures the smooth and reliable power flow over the transmission corridor.

Power systems are viewed as complex, non-linear, dynamical system. The networks of power system is open to large disturbances, faults, switching of lines and low frequency power oscillations which hamper the transientstability and first swing stability of the system and eventually results in voltage collapse.

Stability of system depends on mainly three factors.

1) voltage

2) phase angle

3) frequency

Due to continuously varying load from hour to hour there is a huge variation in voltage.It is quite troublesome to cope-up with varying demand in minimum time. Due to fault in line,electrical power is reduced but mechanical power remains constant.Therefore the turbine delivers extra energy to alternator.Once the fault is sorted out, alternator transfers this excess energy to the network so as to maintain first swing stability.

It is very difficult to enhance the excess energy capability of alternator dynamically.Therefore FACTS devices like SSSC,SVC,STATCOM etc. are incorporated.FACTS devices are more effective in power oscillation damping.

Transient stability of a system refers to its capability of being stable when subjected to large disturbances such as instant faults, loss of generation or tripping of lines. This type of stability depends on the initial operation condition of the system and the severity of the disturbance as well. For any specified disturbance, there is always an associated power transference limit, called the transient stability limit, above which the system will become dynamically unstable. Also, the transient stability limit is always below the steady-state stability limit [4]. It may have many different values, depending upon the nature and magnitude of the disturbance. 
The factors influencing the transient stability of power system are the transmission line reactance reactive power absorptions \& injection.[5]. Since, transient stability of power system is directly related to the power flow in the transmission corridor and it is considered as a matter of energy balance. The mismatches in power can be well regulated by insertion of series and shunt capacitances by enhancing the power flow in the line [6].In this paper we have also simulated a model and shown the effect of facts device such as svc and series capacitors on the transient stability of power system and how the CCT can be increased so that system will remain stable.

\section{Basic Principle Of Operation Of SVC}

The SVC is the most widely employed FACTS Controller. SVC is shunt connected device which can generate or absorb Reactive power .Output of SVC is adjusted to exchange the capacitive or inductive current such that it can maintain the power system parameters within specified limit (Normally Bus voltage) .Fig. 1 shows the configuration of SVC which is generally a controllable inductor in parallel with a switchable capacitance.

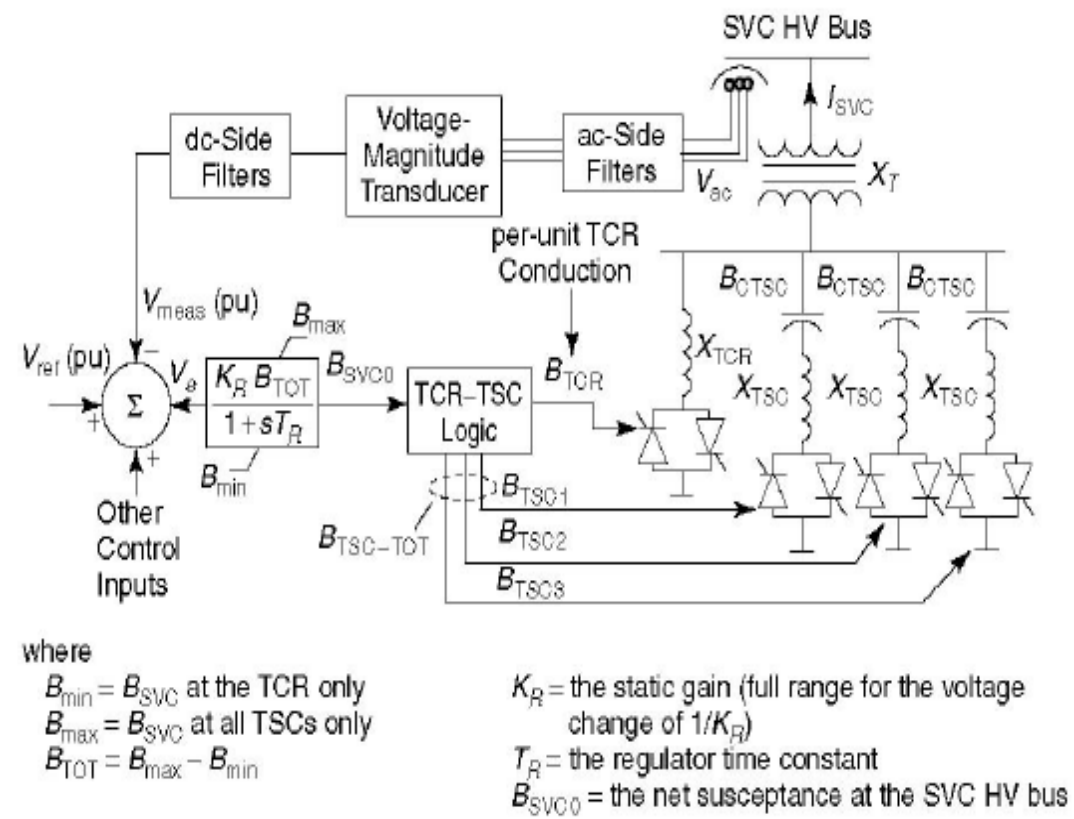

Fig(1) Functional Diagram of SVC

SVC may be a Thyristor controlled reactor (TCR), or thyristor switched capacitor (TSC), best is the combination of both for efficient performance. As shown in figure high voltage side bus voltage is measured and processed and filtered after that compared with the reference voltage and then the error voltage is processed by a gain time constant regulator which provides the desired or required susceptance for the SVC which further is implemented by a control logic which decides how many capacitors to be switched in or out (no of TSCs to be operational) and firing angle of reactors for the desired susceptancevalue[2].

\section{Basic Principle Of Operation Of Statcom}

A STATCOM is a controlled reactive power source. It provides desired reactive power generation as well as absorption purely by means of electronic processing of voltage and current waveforms in a voltage source converter(VSC). A voltage source converter is connected to utility bus through magnetic coupling. As shown in Figure(2) a STATCOM can be seen as an adjustable voltage source behind a reactance. This implies that capacitor banks and shunt reactors are not needed for generation and absorption of reactive power, giving a compact design, a small footprint, as well as low noise and low magnetic impact[2].

Reactive power exchange between the converter and the ac system can be controlled by varying the amplitude of the three-phase output voltage, $E s$, of the converter as illustrated in Figure (2). If the amplitude of the output voltage is increased above that of the utility bus voltage, Et, then a current flows, through the reactance, from the converter to the ac system, and the converter generates capacitive reactive power for the ac system. If the amplitude of the output voltage is decreased below the utility bus voltage, then the current flows from the ac system to the converter, and the converter absorbs inductive reactive power from the ac system. If the output voltage equals the ac system voltage, the reactive power exchange becomes zero, and the STATCOM is said to be in a floating state. 

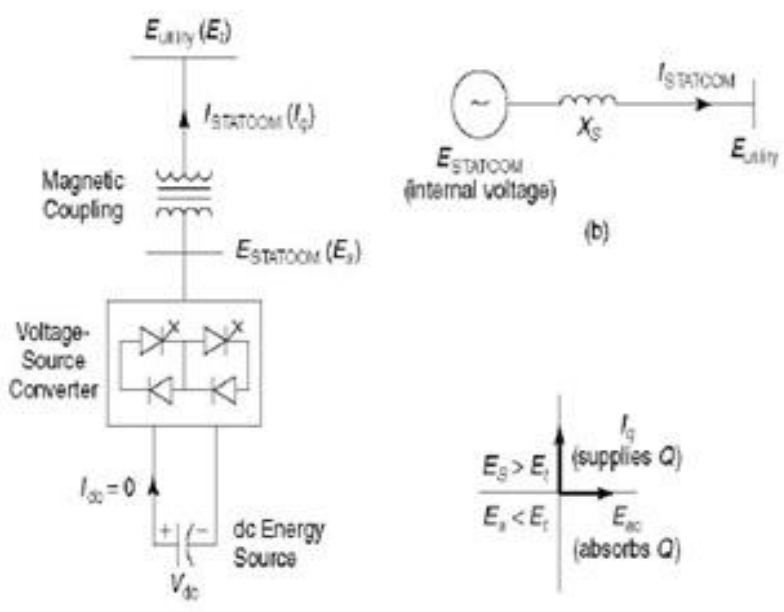

(b)

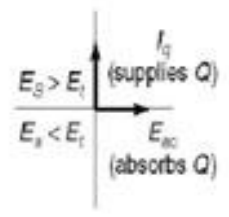

Fig(2) Basic STATCOM Principle Diagram

Adjusting the phase shift between the converter output voltage and the ac system voltage can similarly control real power exchange between the converter and the ac system. That is, the converter can supply real power to the ac system from its dc energy storage if the converter output voltage is made to lead the ac system voltage. On the other hand, it can absorb real power from the ac system for the dc system if its voltage lags the ac system voltage.

STATCOM can supply both the capacitive and the inductive compensation and is able to independently control its output current over the rated maximum capacitive or inductive range irrespective of the amount of the ac system voltage. This is particularly useful in situations where the STATCOM is needed to support the system voltage during and after faults where voltage collapse would otherwise be a limiting factor.

\section{Case Study of IEEE 14 Bus}

\section{Simulation And Results}

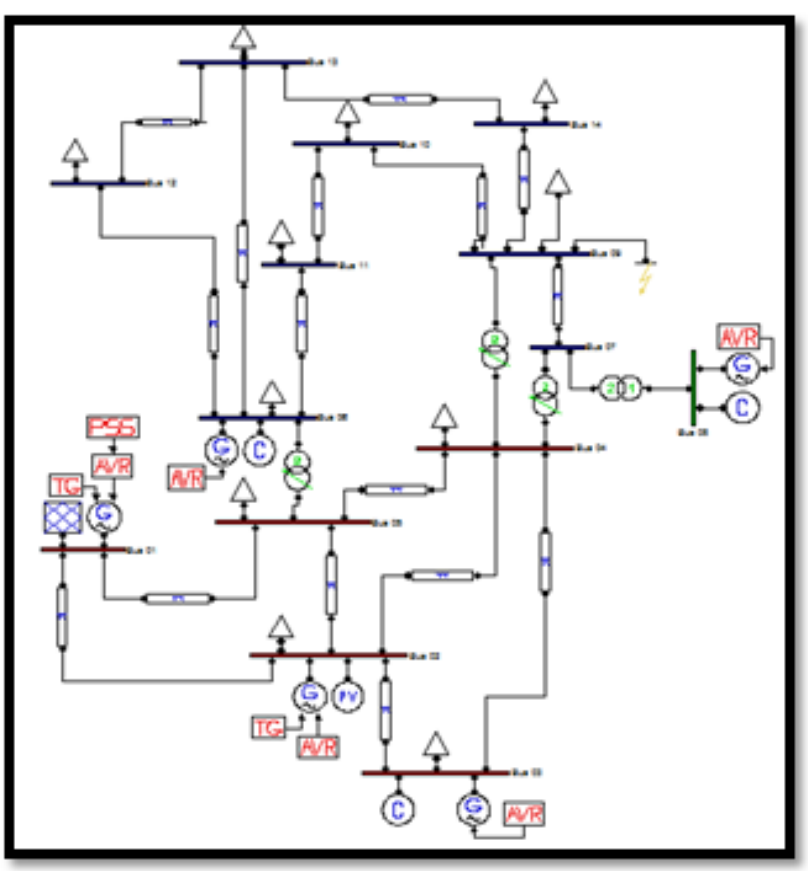

Fig (3) Model of IEEE 14 Bus system

IEEE 14 Bus system is considered for the study purpose shown in Fig(3) which consist of five generators respectively placed at Bus 1, Bus 2 , Bus 3 , Bus 6 , Bus 8 , in which Generator 1 placed at Bus 1 is equipped with both AVR and PSS while rest of the Generators are equipped with AVR only.

A three phase bus fault is introduced into the system to observe the response of the system and behavior of machine .Fault is introduced at Bus 9 after 0.2 seconds and persisted up to 0.05 seconds and then got cleared . 
In Fig (4) Speed deviation of three synchronous machines placed respectively at Bus 1, Bus 2, Bus 3 are shown, which clearly shows that synchronous generator 3 which is equipped only with AVR and fast exciter so the generator is able to maintain First swing stability but may lose the synchronism during second swing but with the addition of a PSS contributes to the damping of second and subsequent swings .

As can be seen from Fig (4) wsyn3 although first swing is limited but second swing is growing in nature and depending on the nature of fault these oscillation may aggravate other modes of oscillation and may result in some generators going out of step leading to a collapse. While wsyn1 only First swing is present and having slightly higher amplitude than in case of $\omega$ syn3 (with AVR only) but second swing and subsequent swings are damped efficiently.

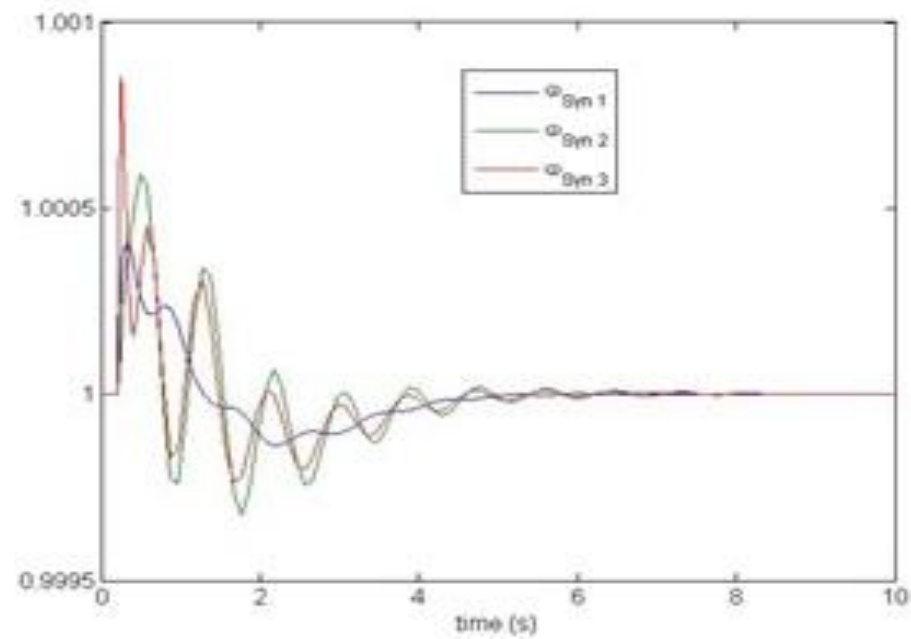

Fig(4) Speed variations of Synchronous Generators with PSS.

Same system response with fault at same bus when observed without PSS as shown in Fig (5) the damping time was very high 11 seconds and the magnitudes of swings were also high which can lead to the damage of generators, generator may go out of step leading to a cascaded event .So contribution of PSS can be clearly identified .

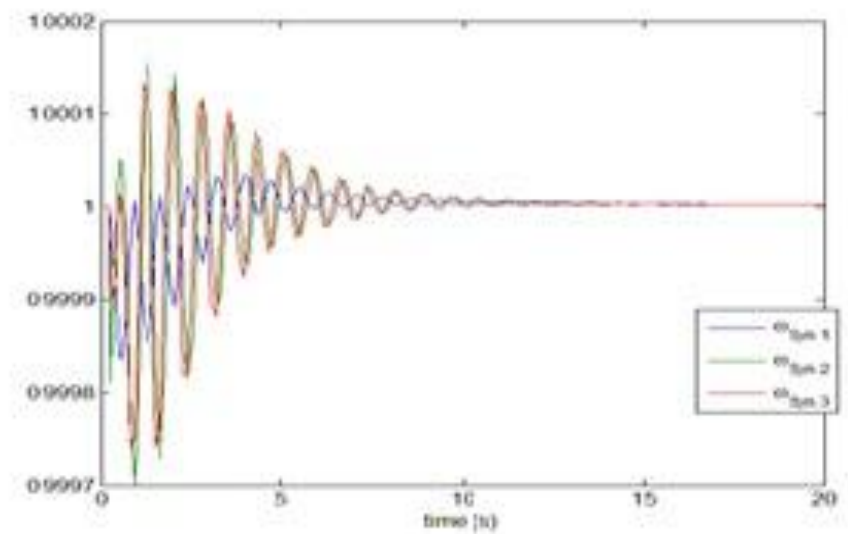

Fig(5) Speed variations of Synchronous Generators without PSS.

\section{(B) Case Study of IEEE 14 Bus With Statcom}

IEEE 14 bus is simulated with the STATCOM placed at Bus -2 as shown in fig(7) and with a 3 phase fault at Bus -9 , all the three generators speed variation is observed as shown in Fig(8). It is clearly shown in Fig 8) that the amplitude of speed variation of generators are minimized well and also the oscillations are well damped within limits although the Generator 2, 3 are not equipped with PSS,a much more effective damping can be achieved with all the generators equipped with PSS.

Amplitude of First swing is also limited and amplitude of initial oscillation is minimized. Another important aspect is that Damping time of oscillation is much less with STATCOM ,in fig (8) only 4 seconds than in previous case as seen in fig $(5 \& 4)$ was 11 and 6 seconds, so STATCOM also provides quick damping, which is must require feature in a complex power system to avoid the machines from losing their synchronism and to avoid disturbances. As oscillations may limit the line loadabilty and may create congestion in the network. 


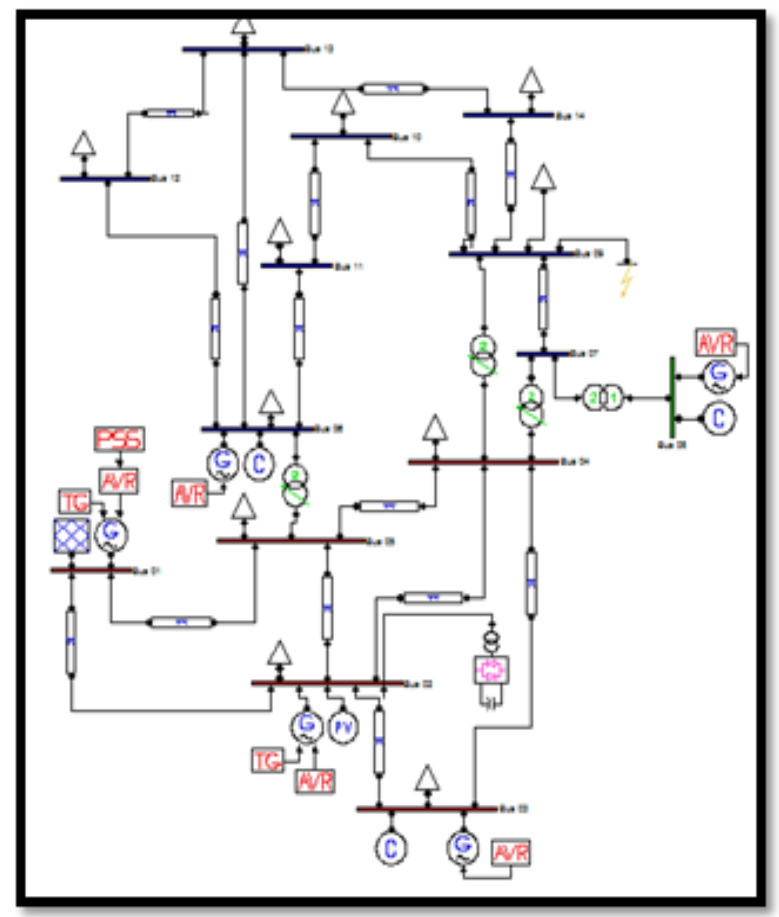

Figure(7) IEEE 14 Bus with STATCOM at Bus-2

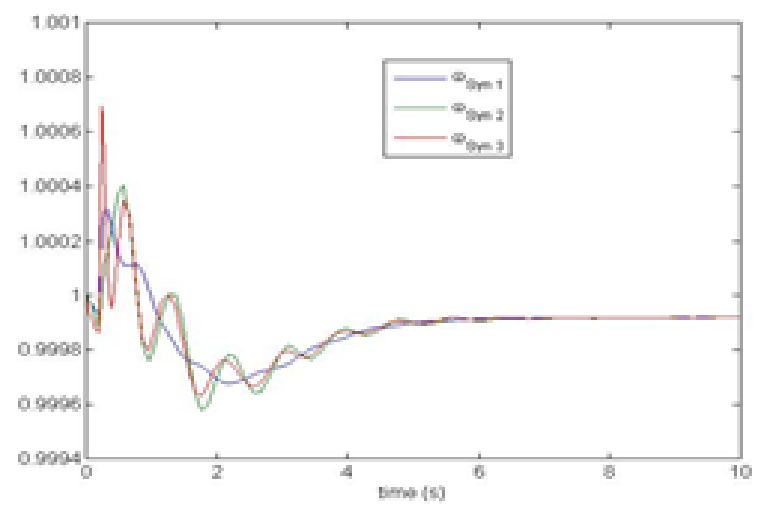

Figure(8) Speed variations of Synchronous Generators with STATCOM at Bus -2.

Same simulation is performed with the SVC used instead of STATCOM and speed deviations are observed as shown in fig (9), Speed deviation plots are almost same as with STATCOM as the IEEE 14 bus itself is not that much complicated but with a different kind of fault and in a larger system they may behave differently .So to observe the overall system stability eigenvalue analysis needs to be done which is described in next section.

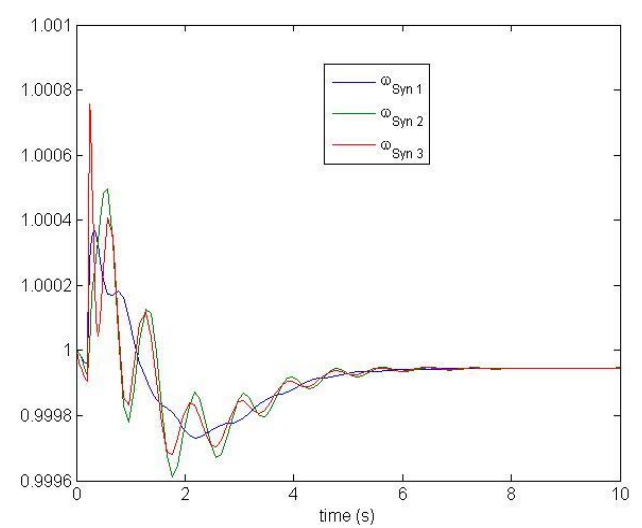

Fig (9) Speed variations of Synchronous Generators with SVC at Bus -2. 


\section{Eigen Value Assessment}

In order to identify oscillatory modes of a multi-machine system, the linearized system model including PSS and FACTS devices system can be used by

$\Delta X=A \Delta X+B$
$\Delta Y=C \Delta X+D \Delta U$

Where

$\Delta \mathrm{X}$ is the state vector of length equal to the numbers of states $\mathrm{n}$.

$\Delta \mathrm{Y}$ is the output vector of length $\mathrm{m}$.

$\Delta \mathrm{U}$ is the input vector of length $\mathrm{r}$.

$\mathrm{A}$ is the $\mathrm{n}$ by $\mathrm{n}$ state matrix.

$\mathrm{B}$ is the control or input matrix of size $\mathrm{n}$ by $\mathrm{r}$.

$\mathrm{C}$ is the output matrix of size $\mathrm{m}$ by $\mathrm{n}$.

$\mathrm{D}$ is the feed forward matrix of dimensions $\mathrm{m}$ by $\mathrm{r}$.

The equation

$\operatorname{det}(\lambda \mathrm{I}-\mathrm{A})=0$

is referred to as the characteristic equation of matrix $A$ and the values of $\lambda$, which satisfy the characteristic equation, are the eigenvalues of matrix A. Because the matrix $A$ is a $n$ by $n$ matrix, it has $n$ solutions of eigenvalues $\lambda$.

For a particular eigenvalue, the real part of the eigenvalue gives the damping, and the imaginary part gives the frequency of oscillation. The relative damping ratio is given by

$$
\varepsilon=\frac{-\sigma}{\sqrt{\sigma^{2}+\omega^{2}}}
$$

\section{(A) Case Study: Eigen values with Statcom}

IEEE 14 bus is simulated in same environment with the fault at Bus 9 and STATACOM placed at Bus-2 and eigenvalues of the systems are plotted as shown in Fig(10).

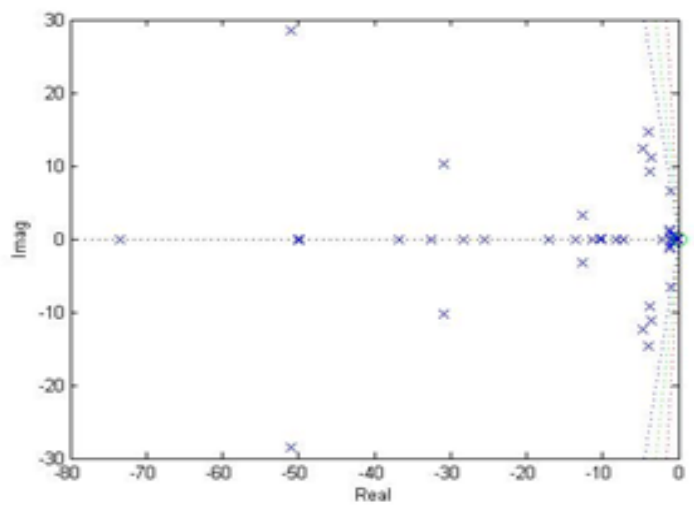

Fig(10) Eigenvalues plot with STATCOM

(B) Case Study: Eigen values with Svc

IEEE 14 bus is simulated in the same environment with the fault at Bus 9 and SVC placed at Bus-2 and eigenvalues of the systems are plotted as shown in Fig(10).

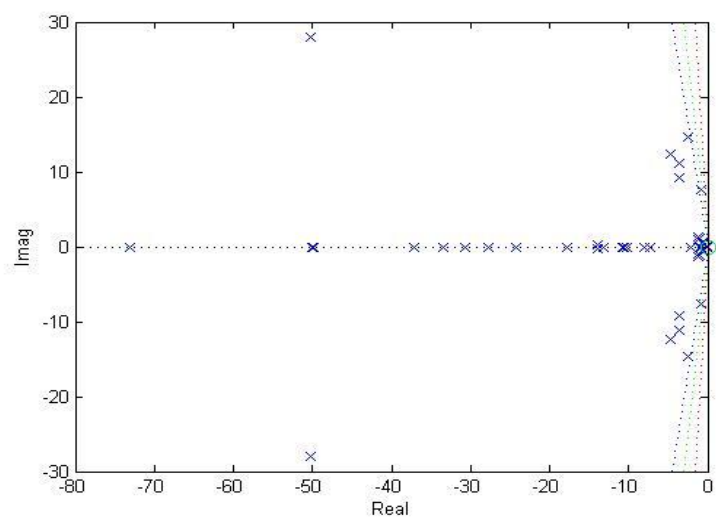

Fig(11) Eigenvalues plot with SVC 
STATCOM enhances the overall system stability which can be understood by eigen values of the system, it tries to shift the eigenvalues in the left half plane which in lieu creates more stability margin for the system and it also improves the stability of system hence improving the damping of the system. Although SVC is also damping the oscillations properly as shown in fig(9) but STATCOM also improves the overall system stability. As shown in Table 1, some of the eigenvalues are shown and compared with SVC and STATCOM which clearly shows that with STATCOM it is trying to shift the eigenvalues to the left half plane.

\begin{tabular}{|l|l|l|}
\hline Most associated states & Eigenvalues With STATCOM & Eigenvalues With SVC \\
\hline vr1_Exc_1, e2q_Syn_1 & $-52.2+28.20$ & $-50.01+28.2$ \\
\hline omega_Syn_5, delta_Syn_5 & $-4.65+12.40$ & $-4.60+12.40$ \\
\hline omega_Syn_2, delta_Syn_2 & $-3.71+11.11$ & $-3.52+11.11$ \\
\hline
\end{tabular}

Table(1) Comparison of eigenvalues

Thus STATCOM is having advantage over SVC as it also improves the overall system stability and enhances and aids positive damping to the system other advantages of the STATCOM are that they can be integrated with renewable for stabilizing and smoothening the output of renewable as discussed in the next section.

\section{Utilisation of Statcom for Renewable Integration}

With the proliferating renewable energy penetration in the electric grids the power quality(PQ)is adversely affected .Issues related to $\mathrm{PQ}$ generally include power frequency disturbances due to low frequency phenomenon or voltage sag/ swell, power system transient, low power factor, electrostatic discharges, electromagnetic interferences due to high frequency phenomenon, switching of loads and majorly due to system harmonics .etc. Long transmission lines are more vulnerable to PQ issues. So to eliminate such problems stress has been given on distributed generation(DG) with integration of renewable energy systems into grid. To facilitate improved PQ custom power devices (CPD) are incorporated like STATCOM etc.

\section{Mitigation of PQ Problems}

PQ problems can be eradicated either from generation side or from load side. Load conditioning is done to ensure that the components of system are resistant to power disturbances and can work smoothly on voltage distortions. To suppress the line disturbances, line conditioning systems are developed like CPD.STATCOM is specially designed for power factor correction, to balance load, filters to suppress current harmonics and for voltage regulation. STATCOM comprises voltage or current source, PWM converter. It generates 180 degree phase shift harmonic components to compensate current harmonics and it improves power factor. As it controls only reactive power supply. Its application gets reduced .It has no abilityto control active power. Since renewable energy resources are dependent on environmental conditions, there is fluctuation in power which can't be smoothened alone by STATCOM. So the STATCOM is provided with battery energy storage system(BESS).So the whole system has both active and reactive power control stability. STATCOM with BESS enhances utilization factor and maintains grid stability.

\section{Case Study Of Wind Generator And Impact Of Statcom}

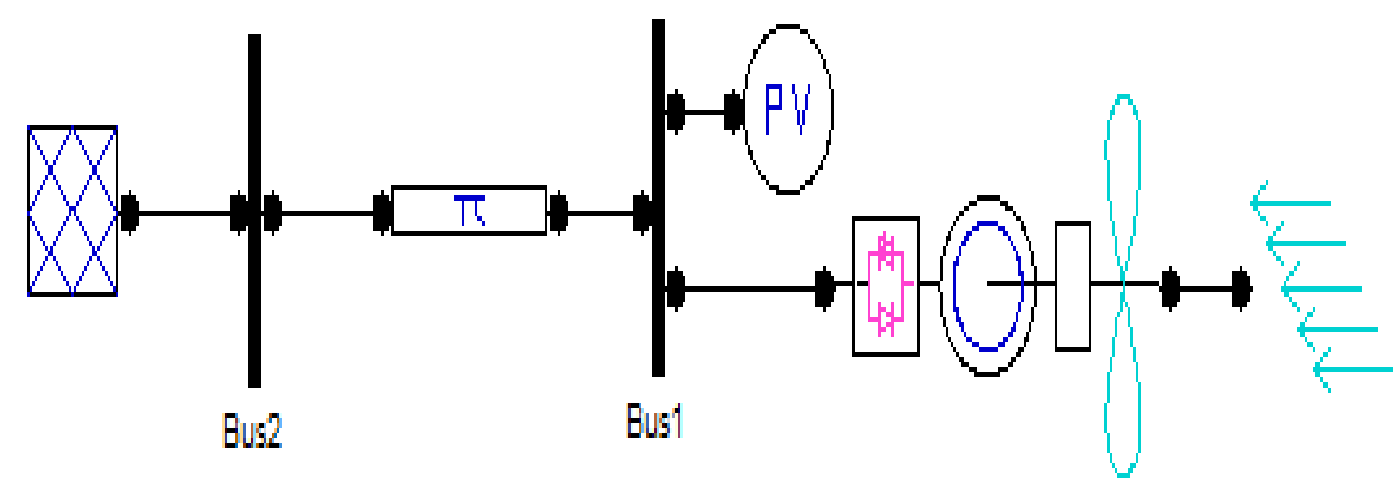

Fig(12) Variable speed wind turbine with direct drive synchronous generator.

As shown in fig(12) wind generator is simulated to observe the effect on Bus voltage and bus voltage of Bus 1 is observed with and withoutSTATCOM. In fig(13) Bus voltage of Bus-1 plotted where fluctuation in voltage is observed . 


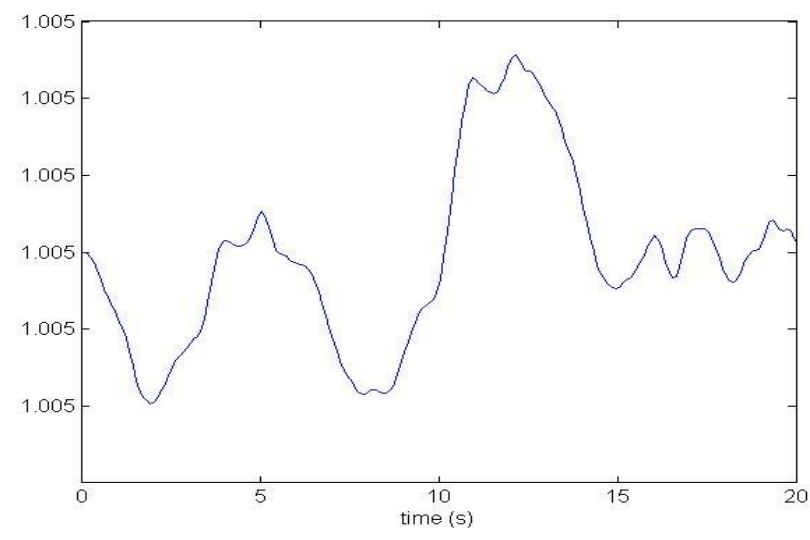

Fig(13) Plot of Bus-1 voltage without STATCOM

Same case is simulated and STATCOMplaced at Bus-1,and Bus-1voltage is plotted where as can be seen in Fig(14) the voltage settled at $1.07 \mathrm{Pu}$ without any fluctuation in Voltage, so integration of STATCOM with renewable helps in improving and maintain the voltage as discussed in above section.

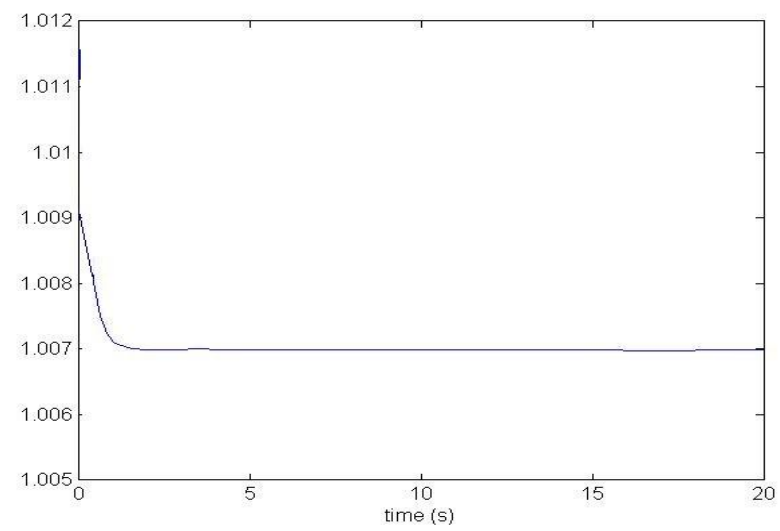

Fig(14) Plot of Bus-1 voltage with STATCOM

Integration of STATCOM does not only improves the voltage profile and supports the reactive power requirement but also enhances the overall stability by improving voltage stability and increasing the stability margin. As discussed, it also mitigates the problem of PQ when interconnected with grid.

\section{Conclusion}

The paper showcases the FACTS controller based scheme to mitigate escalating power oscillation issues and discusses about effective methods of its damping by using SVC and STATCOM.Different cases has been studied through the simulation of IEEE 14 bus System. Importance of AVR and PSS for maintaining first swing stability and damping consecutive swings respectively has been mentioned. STATCOM is advantageous over SVC as it improves the overall system stability by shifting the eigen values in left half plane .Large renewable energy penetration in electric grids results in deterioration of power quality. Power electronic devices like BESS based STATCOM is very much useful for smooth and reliable operation with integration of renewable with grid which helps in improving utilization factor.

\section{References}

[1]. N.G.Hingorani and L.Gyugyi” Understanding FACTS Concepts and Technology of Flexible AC Transmission Systems," IEEE Press, New York, 2000.

[2]. Varma, Rajiv K. "Introduction to FACTs controllers." 2009 IEEE/PES Power Systems Conference and Exposition. 2009.

[3]. P. Kundur: "Power system stability and control" (McGraw- Hill, 1994), pp. 1103-127.

[4]. Edward Wilson Kimbark" Power system stability, volume 1," IEEE Press, 445 Hoes Lane Piscataway, NJ 08855, 1995, A John Wiley \& sons, inc., publication, Hoboken, New Jersey.

[5]. SadikoviC, Rusejla. "Use of FACTS devices for powerflow control and damping of oscillations in powersystems." PhD diss., SWISS FEDERAL INSTITUTEOF TECHNOLOGY ZURICH, $2006 .$.

[6]. Anderson, P.M, Fouad, A.A, "Power System Control and Stability" Iowa state university press, $1977 .$.

[7]. Yao Nan Yu.”Electric Power System”Academic Press, 1983. 